\title{
Automated Parameterization of Active Contours: A Brief Survey
}

\author{
E.A. Mylona, M.A. Savelonas, D. Maroulis \\ Department of Informatics and Telecommunications, \\ University of Athens, Panepistimiopolis, Ilissia, 15703, Athens, Greece \\ \{emylona, msavel, dmaroulis\}@di.uoa.gr
}

\begin{abstract}
Active contours yield segmentation results which depend on an initial empirical parameterization stage. The latter is a tedious and time-consuming process that requires technical skills from the end user. Automated adjustment of active contour parameters is still a challenging issue. This survey reviews stateof-the-art active contours which attempt to cope with the issue of empirical parameterization, so as to secure the objectivity and robustness of the segmentation results. Numerous attempts utilize information associated with contour evolution and shape priors, whereas others are hybrid, driven by both empirical and automatically obtained parameter settings. Most recent models are spatially varying and versatile regarding the application and the energy functional to be minimized.
\end{abstract}

Keywords-Active Contours, Automated Parameter Adjustment, Segmentation.

\section{INTRODUCTION}

Empirical parameterization of active contours [1]-[8] is a laborious process which requires considerable time to be performed as well as technical skills from the end user. Moreover, the obtained segmentation results are highly subjective and reproducibility is complicated. Even though in most cases the segmentation quality is acceptable, it is controversial since it relies on the technical skills of the end user.

Although a generally applicable framework for automated parameter adjustment that eliminates heuristic considerations is yet to be developed in active contour research, several approaches address this issue. Such approaches are of crucial importance since they are potentially capable of relieving users from the trial and error process as well as to endow segmentation results with objectivity and robustness.

Region-based active contours are widely applied on image segmentation due to their inherent noise-filtering mechanism and their topological adaptability. Furthermore, they are robust to weak edges and intensity inhomogeneity [9]-[13]. They are guided by the minimization of an energy functional $E_{\text {total }}$ described as follows:
$E_{\text {total }}=w_{\text {reg }} \cdot E_{\text {reg }}+w_{d f} \cdot E_{d f}$

where $E_{r e g}$ and $E_{d f}$ are regularization and data fidelity energy terms, respectively, whereas $w_{\text {reg }}$ and $w_{d f}$ are the corresponding weighting parameters which are typically adjusted in an empirical manner.

This survey reviews active contour parameter adjustment approaches, which aim to confront the challenging issue of automated parameterization. These approaches are classified according to the information utilized in the computation of parameters. To the best of our knowledge, such a review is missing in active contour literature.

\section{AUTOMATIC ACTIVE CONTOUR MODELS}

\section{A. Time-Dependent Active Contour Parameterization}

Tsai et al. [14] proposed a region-based, shape-driven active contour model for medical image segmentation by applying principal component analysis to a collection of signed distance representations of the training data. According to this model, parameters are calculated by means of the gradient descent algorithm which minimizes an energy functional as follows:

$w^{t+1}=w^{t}-a_{w} \cdot \nabla w E$
$p^{t+1}=p^{t}-a_{p} \cdot \nabla p E$

where $w=\left\{w_{1}, w_{2}, \ldots w_{k}\right\}$ are the shape weights for the $k$ eigenshapes, $\quad p=\left\{p_{1}, p_{2}, \ldots p_{n}\right\}$ pose parameters used to jointly align the $n$ binary images, $a_{w}, a_{p}$ positive step-size parameters and $E$ the energy functional. The model is capable of embedding various region-based energy functionals to be minimized via the gradient descent algorithm and shape and pose parameters are updated dynamically within the iterative procedure of active contour evolution. However, cases of erroneous behavior of the 
active contour in the early evolution stages have not been encountered. Figure 1 depicts the block diagram of the pipeline of Tsai et al. model [14].

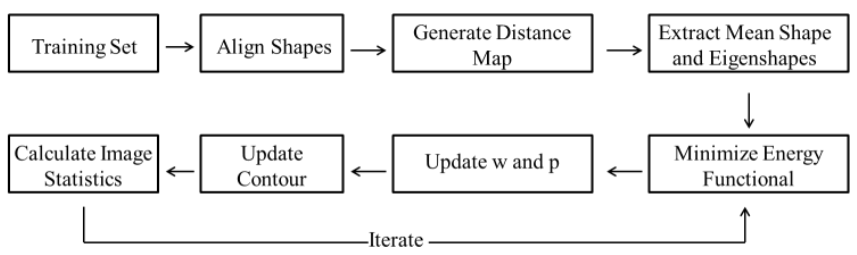

Fig. 1. Block diagram of the pipeline of Tsai et al. model [14].

Pluempitiwiriyawej et al. [15] proposed a stochastic active contour scheme for automated segmentation of cardiac MR images. The energy functional that is minimized combines both region-based and edge-based information by utilizing shape priors of the heart and is defined as follows:

$$
\mathrm{E}(\varphi)=\lambda_{1} \cdot \mathrm{E}_{1}(\varphi)+\lambda_{2} \cdot \mathrm{E}_{2}(\varphi)+\lambda_{3} \cdot \mathrm{E}_{3}(\varphi)+\lambda_{4} \cdot \mathrm{E}_{4}(\varphi)
$$

where the first, second, third and fourth term incorporates region-based, edge-based, shape prior information and smoothness regulation, respectively and are described according to the following:

$$
\begin{aligned}
\mathrm{E}_{1}(\varphi) & =\int_{\Omega}-\ln \left[p_{1}(I(x, y))\right] \cdot H(\varphi(x, y)) \\
& -\ln \left[p_{2}(I(x, y))\right] \cdot[1-H(\varphi(x, y))] d x d y \\
\mathrm{E}_{2}(\varphi) & =\int_{\Omega}-\left|\nabla G_{\sigma} * I(x, y)\right| \cdot \mid \nabla H(\varphi(x, y)) d x d y \\
\mathrm{E}_{3}(\varphi) & =\int_{\Omega} D^{2}(x, y) \cdot \delta(\varphi(x, y)) \cdot|\nabla \varphi(x, y)| d x d y \\
\mathrm{E}_{4}(\varphi) & =\int_{\Omega} \delta(\varphi(x, y)) \cdot|\nabla \varphi(x, y)| d x d y
\end{aligned}
$$

where $p_{1}, p_{2}$ are the assumed pdfs inside and outside the contour, $G_{\sigma}$ the two-dimensional Gaussian kernel with variance $\sigma^{2}, D^{2}$ the ellipse distance function and $\delta$ the Dirac function. Parameters $\lambda_{1}, \lambda_{2}, \lambda_{3}, \lambda_{4}$ control the strength of each of the terms $E_{1}, E_{2}, E_{3}, E_{4}$, respectively. Aiming at automated parameter adjustment, the authors present an annealing algorithm through which parameters adapt their values as the segmentation evolves. The selected annealing schedules are defined as follows:

$$
\lambda_{1}(n)=\lambda_{1}(1)-\frac{n\left[\lambda_{1}(1)-\lambda_{1}(N)\right]}{N}
$$

$$
\begin{aligned}
& \lambda_{2}(n)=\frac{1}{2}\left[\lambda_{2}(1)-\lambda_{2}(N)\right] \cdot\left[1+\cos \left(\frac{n \pi}{N}\right)\right]+\lambda_{2}(N) \\
& \lambda_{3}(n)=\frac{\lambda_{3}(N)-\lambda_{3}(1)}{\cosh \left[10\left(\frac{n \pi}{N}-1\right)\right]}+\lambda_{3}(1)
\end{aligned}
$$

where $n$ is the iteration number, $N$ the total number of iterations and $\lambda_{i}(1), \lambda_{i}(N)$ the initial and final values of $\lambda_{i}$, respectively. It should be noted that $\lambda_{4}$ is selected to be constant. Similar to the model of Tsai et al. [14], parameters are updated during contour evolution. Hence, errors in the early stages of evolution will propagate to the later stages of contour convergence.

\section{B. Active Contour Parameterization using Shape Priors}

Keuper et al. [16] proposed a probability-based method for dynamic adjustment of active surface parameters for the segmentation of cell nuclei. The method utilizes the total energy of an active surface defined as:

$$
E(X)=a \cdot E_{\mathrm{int}}(X)+b \cdot E_{\text {ext }}(X)
$$

where $X$ is the function of the active surface, $E_{\text {int }} E_{e x t}$ the internal and external energies, respectively and $a, b$ weighting parameters. The method is based on 2-spherelike objects of gray value profiles $r_{x_{i}} \in R^{l}$ of surface vertices $v_{i}$ in radial direction, where $l$ is the number of sampling points on the profile. The weighting parameters which are assigned to vertices $v_{i}$ are calculated as follows:

$a_{i}=1-p\left(B \mid r_{x_{i}^{e t t}}\right), \quad b_{i}=p\left(B \mid r_{x_{i}^{e x t}}\right)$

$p\left(B \mid r_{x_{i}^{e x t}}\right)=\frac{p\left(r_{x_{i}^{e x t}} \mid B\right) \cdot P(B)}{P\left(r_{x_{i}^{e x t}}\right)}$

where $P(B)$ is the only high level parameter. The method is beneficial compared to standard active surfaces with constant weighting parameters, yet it is primarily dependent on the shape of the target region.

Liu et al. [17] proposed a localized active contour model for lip contour extraction in which proper parameters are automatically selected. The proposed approach utilizes a minimum-bounding ellipse in order to initialize the evolving curve. The final energy functional to be minimized is given as follows: 


$$
\begin{aligned}
E(\varphi) & =\int_{\Omega_{v}} \delta(\varphi(u)) \cdot \int_{\Omega_{v}} \mathrm{~B}(u, v) \cdot F(I(v), \varphi(v)) d v d u \\
& +\lambda \cdot \int_{\Omega_{v}} \delta(\varphi(u)) \cdot\|\nabla(u)\| d u
\end{aligned}
$$

where $B(u, v)$ is a characteristic function, $F$ is a localized region-based force formed by substituting local means for global ones described as:

$F=-\left(\mu_{\text {in }}(u)-\mu_{\text {out }}(u)\right)^{2}$

and $\lambda$ a weighting parameter. The level set is initialized as a minimum-bounding ellipse defined as:

$$
\frac{X^{2}}{r_{a}^{2}}+\frac{Y^{2}}{r_{b}^{2}}=1
$$

$X=\left(x-x_{c}\right) \cdot \cos \theta+\left(y-y_{c}\right) \cdot \sin \theta$

$Y=\left(y-y_{c}\right) \cdot \cos \theta-\left(x-x_{c}\right) \cdot \sin \theta$

$r_{a}=\frac{1}{2}\left(\left(L b_{x}-L a_{x}\right)^{2}+\left(L b_{y}-L a_{y}\right)^{2}\right)^{\frac{1}{2}}$

$$
r_{b}=\frac{1}{2}\left(\left(V b_{x}-V a_{x}\right)^{2}+\left(V b_{y}-V a_{y}\right)^{2}\right)^{\frac{1}{2}}
$$

where $\left(x_{c}, y_{c}\right)$ is the origin center of the minimumbounding ellipse, $\theta$ the inclined angle defined to be positive in the counter-clockwise direction and $L_{a}, L_{b}, V_{a}, V_{b}$ the left, right, upper, lower corner, respectively. The parameters of the ellipse are selected automatically; however $\lambda$ is still empirically determined. Additionally, the proposed approach hinges on the shape of the target region.

\section{Hybrid Active Contour Parameterization using both Empirical and Automatically obtained Parameter Settings}

Hsu et al. [18] proposed an active contour model called Poisson Gradient Vector Flow (PGVF) which employs a genetic algorithm for automated segmentation of liver PET images. The original liver PET image is imported and its edge map is generated by Canny edge detection. The parameters of the latter are calculated by the design of the fitness function, which is defined as follows:

$$
F=a \cdot \frac{\max (L)}{\min (N)}
$$

where $L, N$ are the length and total pixel number of the contour line obtained by the binary edge map, which is detected by Canny edge detection, and $a$ a weighting number.

Yushkevish et al. [19] developed an open source active contour-based application, called ITK-SNAP, for automated segmentation of medical images by clinical users, who are unfamiliar with the intrinsic mechanisms of active contour algorithms. ITK-SNAP implements the three-dimensional geodesic active contour model written as:

$$
C_{t}(t, u)=\left(a \cdot g_{I}+b \cdot k \cdot g_{I}+\gamma \cdot \nabla g_{I} \cdot N\right) \cdot N
$$

where $C_{t}(t, u)$ is the contour at time $t$ parameterized by $u, k$ the mean curvature of the contour, $N$ the contour normal, $g_{I}$ a function that decreases monotonically with the gradient magnitude of image $I$ and $a, b, \gamma$ modulating weights. To assist the user in setting these parameters, ITKSNAP employs a structured wizard segmentation workflow and feedback mechanisms. Although performed in a more intuitive manner, parameter settings are still empirically determined.

\section{Machine Learning and Spatially Varying Models}

Iakovidis et al. [20] proposed a framework for thyroid ultrasound image segmentation which encompasses an automatic parameter tuning mechanism based on genetic algorithms. The proposed framework implements the Variable Background Active Contour (VBAC) model [21], which extends the energy functional of the Chan-Vese model [9] by defining the difference $D(\varphi(x, y))$ as follows:

$D(\varphi(x, y))=H(\varphi(x, y)+a)-H(\varphi(x, y))$

where $a$ is a positive constant. Parameters $w_{r e g}, w_{d f}$ and $a$ are automatically adjusted through a genetic optimization problem. Parameters are encoded into a single bit-string, called chromosome. Their values are constrained within discrete, worst-case ranges. The minimum and maximum values of these ranges as well as the sampling rate considered are experimentally determined. This heuristic approach converges slowly in locally optimal solutions.

Allili et al. [22] proposed an automatic estimation of the weighting parameters for segmentation combining boundary and region-based information. Boundary information is formulated using a multi-band edge detector, whereas region-based information is formulated using a mixture of Gaussians. Let $U=\left(u_{1}, u_{2}, \ldots u_{n}\right)$ be a multi-valued image defined on $\Omega$ and $\Omega_{k}, \partial \Omega_{k}$ are the region and its boundaries, respectively. The energy functional that is minimized is 
described as follows:

$$
\begin{aligned}
& E\left(\bigcup_{k=1}^{M} \partial \Omega_{k}(s), \Theta\right)=a \cdot E_{b}+b \cdot E_{r} \\
& E_{b}=\sum_{k=1}^{M} \oint_{\partial \Omega_{k}} g\left(\nabla I\left(\partial \Omega_{k}(s)\right)\right) d s \\
& E_{r}=\sum_{k=1}^{M} \iint_{\Omega_{k}}-\log \left[t_{k}(x)\right] d x d y
\end{aligned}
$$

where $M$ is the number of regions, $\Theta$ the mixture of parameters $\theta_{k}$ of each pdf, $t_{k}=p\left(\theta_{k} \mid U(x)\right.$ and $a, b$ weighting parameters. According to this method, the latter establish adaptive values as follows:

$$
\begin{aligned}
& a \propto\left[\widetilde{E}_{b}(x)\right]^{-1}, \quad b \propto\left[\widetilde{E}_{r}(x)\right]^{-1} \\
& \widetilde{E}_{b}(x)=\frac{1}{N} \sum_{(u) \in W(x)} E_{b}(u), \quad \widetilde{E}_{r}(x)=\frac{1}{N} \sum_{(u) \in W(x)} E_{r}(u)
\end{aligned}
$$

where $W(x)$ is a square window which surrounds pixel $x$. This method provides better convergence and robustness to oversegmentation compared to the one utilizing fixed parameters.

Mylona et al. [23] proposed a framework for selfadjustment of region-based active contours, based on texture cues. The latter are mined by filtering methods characterized by multi-resolution, anisotropy, localization and directionality. This information is encoded by entropy-based image "heatmaps", which are able to weight the regularization and data fidelity terms appearing in the region-based energy functional. Parameters are spatially varying, so as to reflect regional image features, and are calculated as follows:

$$
w_{r e g}=\left(1 / w_{d f}\right) \times N \times M, \quad w_{d f}=\arg _{I j k} \max \left(I E_{j k}\left(I_{j k}\right)\right)
$$

where $N, M$ are the rows and columns of the input image, respectively, $I_{j k}$ the band-pass directional subband obtained by the contourlet transform [24] representing the local feature space and $I E_{j k}$ the information entropy of the $k^{\text {th }}$ direction in the $j^{\text {th }}$ level of the transform defined by:

$$
I E_{j k}=-\sum_{n=1}^{N_{j k}} \sum_{m=1}^{M_{j k}} p_{j k}(m, n) \cdot \log p_{j k}(m, n)
$$

$$
p_{j k}(m, n)=\frac{\left|I_{j k}(m, n)\right|^{2}}{\sqrt{\sum_{n=1}^{N_{j k}} \sum_{m=1}^{M_{j k}}\left[I_{j k}(m, n)\right]^{2}}}
$$

The framework of Mylona et al. can be applied to various image modalities, such as biomedical, textured and realworld images and does not require a priori knowledge on the shape of the target region. Moreover, it is versatile i.e. can be embedded in various active contour variations.

\section{CONCLUSIONS}

This survey reviews current active contour parameterization approaches. Their key objective is to relieve domain users from the cumbersome process of empirical parameterization, as well as from extensive developer support, endowing segmentation results with robustness and reproducibility.

The existing automated parameterization approaches attempt to adjust either weighting parameters navigating contour evolution or other types of parameters, which come with supporting algorithms, such as Canny edge detection or Gaussian mixtures, which are integrated into an active contour model.

Several parameterization approaches, as the model of Tsai et al. [14] and Pluempitiwiriyaweg et al. [15], calculate parameters without encountering possible erroneous behavior of the contour in the early evolution stages. Other approaches, including those of Keuper et al. [16] and Liu et al. [17], focus on specific applications and are highly dependent on a priori knowledge, considering the size and the shape of the target region. Alternatives, like the models of Hsu et al. [18] and Yushkevish et al. [19] aim on specific applications, whereas they introduce new fixed parameters.

A number of state-of-the-art parameterization approaches, like those of Ma et al. [25] and McIntosh et al. [26], try to solve the trade-off between energy terms without adjusting each individual parameter separately. Kokkinos et al. [27], Iakovidis et al. [20] and Erdem et al. [28] utilize machine learning algorithms. Nevertheless, these approaches still require technical skills from the end user.

On the other hand, the approaches of Allili et al. [22] and Mylona et al. [23] are spatially varying, reflecting regional image features, and versatile with respect to the application. Additionally, they do not depend on the shape of the target region.

Table 1 summarizes active contour parameter adjustment approaches and compares them with respect to: 1) the level of automation, 2) time-dependency, 3) use of shape priors, 4) spatial variability and 4) use of machine learning. By and large, a 'wish list' of desired features for automated active contour parameterization contains: 1) full automation, 2) time-independency, 3) versatility with respect to the energy 
Table 1. Summary of active contour parameter adjustment approaches

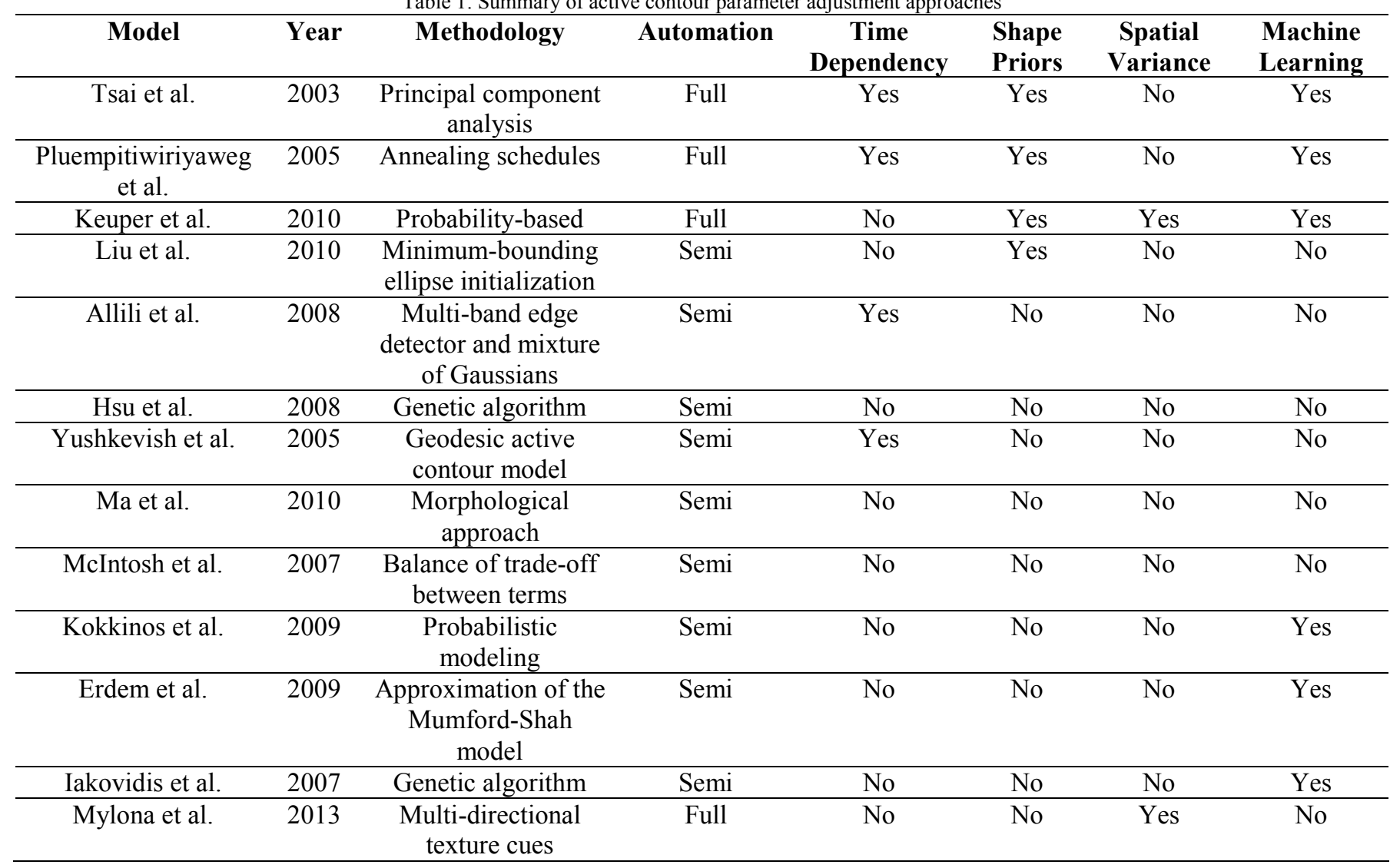

functional, 4) general applicability with respect to the application and the shape of the target region, 5) spatial variability, and 6) user-friendly deprived of developers support.

\section{ACKNOWLEDGEMENT}

This research has been co-financed by the European Union (European Social Fund-ESF) and Greek national funds through the Operational Program "Education and Lifelong Learning" of the National Strategic Reference Framework (NSRF)-Research Funding Program: Heracleitus II. Investing in knowledge society through the European Social Fund.

\section{REFERENCES}

[1] C. Li, C. Xu, C. Gui and M.D. Fox, "Distance Regularized Level Set Evolution and Its Application to Image Segmentation," IEEE Transactions on Image Processing, vol. 19, no. 12, pp. 3243-3254, 2010.

[2] R. Malladi, J.A. Sethian and B.C. Vemuri, "Shape Modeling with Front Propagation: A Level Set Approach," IEEE Transactions on Pattern Analysis and Machine Intelligence, vol. 17, no. 2, pp. 158175, 1995.

[3] N. Paragios and R. Deriche, "Geodesic Active Contours and Level Sets for the Detection and Tracking of Moving Objects," IEEE Transactions on Pattern Analysis and Machine Intelligence, vol. 22, no. 3, pp. 266-280, 2000.
[4] M. Leventon, W. Grimson and O. Faugeras, "Statistical Shape Influence in Geodesic Active Contours," Proc. IEEE Computer Vision and Pattern Recognition (CVPR), vol. 1, pp. 316-323, 2000.

[5] A. Vasilevskiy and K. Siddiqi, "Flux Maximizing Geometric Flows," IEEE Transactions on Pattern Analysis and Machine Intelligence, vol. 24, no. 12, pp. 1565-1578, 2001.

[6] N. Paragios, O. Mellina-Gottardo and V. Ramesh, "Gradient Vector Flow Fast Geometric Active Contours," IEEE Transactions on Pattern Analysis and Machine Intelligence, vol. 26, no. 3, pp. 402407, 2004.

[7] C. Li, C. Xu, C. Gui and M.D. Fox, "Level Set Evolution Without Reinitialization: A New Variational Formulation," Proc. IEEE Computer Vision and Pattern Recognition (CVPR), vol. 1, pp. 430436, 2005.

[8] X. Xie and M. Mirmehdi, "MAC: Magnetostatic Active Contour Model," IEEE Transactions on Pattern Analysis and Machine Intelligence, vol. 30, no. 4, pp. 632-646, 2008.

[9] T.F. Chan and L.A. Vese, "Active Contours Without Edges," IEEE Transactions on Image Processing, vol. 10, no. 2, pp. 266-277, 2001.

[10] X. Bresson, S. Esedoglu, P. Vandergheynst, J. Thiran and S. Osher, "Fast Global Minimization of the Active Contour/Snake Model," Journal of Mathematical Imaging and Vision, vol. 28, no. 2, pp. 151$167,2007$.

[11] A. Tsai, A. Yezzi and A.S. Willsky, "Curve Evolution Implementation of the Mumford-Shah Functional for Image Segmentation, Denoising, Interpolation and Magnification," IEEE Transactions on Image Processing, vol. 10, no. 8, pp. 1169-1186, 2001.

[12] T. Brox and D. Cremers, "On Local Region Models and a Statistical Interpretation of the Piecewise Smooth Mumford-Shah Functional," International Journal of Computer Vision, vol. 84, no. 2, pp. 184-193, 2009. 
[13] E. Strekalovskiy, A. Chambolle, D. Cremers, "A Convex Representation for the Vectorial Mumford-Shah Functional," Proc. IEEE Computer Vision and Pattern Recognition (CVPR), 2012.

[14] A. Tsai, A. Yezzi, W. Wells, C. Tempany, D. Tucker, A. Fan, W.E. Grimson and A. Willsky, "A Shape-Based Approach to the Segmentation of Medical Imagery Using Level Sets," IEEE Transactions on Medical Imaging, vol. 22 no. 2, pp. 137-154, 2003.

[15] C. Pluempitiwiriyawej, J.M.F. Moura, Y.J.L. Wu and C. Ho, "STACS: New Active Contour Scheme for Cardiac MR Image Segmentation," IEEE Transactions on Medical Imaging, vol. 24, no. 5, pp. 593-603, 2005.

[16] M. Keuper, T. Schmidt, J. Padeken, P. Heun, K. Palme, H. Burkhardt and O. Ronneberger, "3D Deformable Surfaces with Locally SelfAdjusting Parameters - A Robust Method to Determine Cell Nucleus Shapes," Proc. IEEE International Conference on Pattern Recognition (ICPR), pp. 2254-2257, 2010.

[17] X. Liu, Y.M. Cheung, M. Li and H. Liu, "A Lip Extraction Method Using Localized Active Contour Model with Automatic Parameter Selection," Proc. IEEE International Conference on Pattern Recognition (ICPR), pp. 4332-4335, 2010.

[18] C.Y. Hsu, C.Y. Liu and C.M. Chen, "Automatic Segmentation of Liver PET Images," Computerized Medical Imaging and Graphics, vol. 32, pp. 601-610, 2008.

[19] P.A. Yushkevich, J. Piven, H. Cody, S. Ho, J.C. Gee and G. Gerig, "User-Guided Level Set Segmentation of Anatomical Structures with ITK - SNAP," Insight Journal, Special Issue on ISC/NAMIC/MICCAI Workshop on Open-Source Software, 2005.

[20] D. Iakovidis, M. Savelonas, S. Karkanis and D. Maroulis, "A Genetically Optimized Level Set Approach to Segmentation of Thyroid Ultrasound Images," Applied Intelligence, Springer - Verlag, vol. 27, no. 3, pp. 193-203, 2007.

[21] D.E. Maroulis, M.A. Savelonas, D.K. Iakovidis, S.A. Karkanis and N. Dimitropoulos, "Variable Background Active Contour Model for Computer-Aided Delineation of Nodules in Thyroid Ultrasound Images", IEEE Transactions on Information Technology in Biomedicine, vol. 11, no. 5, pp. 537-543, 2007.

[22] M. Allili, D. Ziou, "An Approach for Dynamic Combination of Region and Boundary Information in Segmentation," Proc. International Conference on Pattern Recognition (ICPR), pp. 1-4, 2008.

[23] E.A. Mylona, M.A. Savelonas and D. Maroulis, "Self-Adjusted Active Contours Using Multi-Directional Texture Cues," Proc. IEEE International Conference on Image Processing (ICIP), Melbourne, Australia, 2013.

[24] M.N. Do and M. Vetterli, "The Contourlet Transform: An Efficient Directional Multiresolution Image Representation," IEEE Transactions on Image Processing, vol. 14, no. 12, pp. 2091-2106, 2005.

[25] L. Ma and J. Yu, "An Unconstrained Hybrid Active Contour Model for Image Segmentation," IEEE $10^{\text {th }}$ International Conference on Signal Processing (ICSP), pp. 1098-1101, 2010.
[26] C. McIntosh and G. Hamarneh, "Is a Single Energy Functional Sufficient? Adaptive Energy Functionals and Automatic Initialization," in Proc. International Conference on Medical Image Computing and Computer-Assisted Intervention (MICCAI), pp. 503-510, 2007.

[27] I. Kokkinos, G. Evangelopoulos and P. Maragos, "Texture Analysis and Segmentation Using Modulation Features, Generative Models and Weighted Curve Evolution," IEEE Transactions on Pattern Analysis and Machine Intelligence, vol. 31, no. 1, pp. 142-157, 2009.

[28] E. Erdem and S. Tari, "Mumford-Shah Regularizer with Contextual Feedback," Journal of Mathematical Imaging and Vision, vol. 33, pp. 67-84, 2009. 